\title{
El tratamiento de la dimensión social en los estudios de impacto ambiental: Análisis de contenido aplicado al caso de la Comunidad Valenciana / The treatment of the social dimension in environmental impact assessment: A content analysis applied to the case of the Valencian autonomous region
}

\author{
*Guadalupe Ortiz \\ Departamento de Sociología I. Universidad de Alicante. España / Spain \\ Guadalupe.ortiz@ua.es
}

Recibido / Received: 29/06/2017

Aceptado / Accepted: 05/04/2018

\section{RESUMEN}

La demanda de incorporación efectiva de la dimensión social a los procedimientos de Evaluación de Impacto Ambiental tiene un ya largo recorrido tanto desde el ámbito académico de la sociología ambiental como desde sectores públicos y privados. También la legislación vigente reconoce la necesidad de participación ciudadana como principio básico del proceso y exige una atención a las consecuencias que sobre la población tenga el proyecto evaluado. La presente investigación estudia el modo en que esta exigencia se incorpora actualmente en el procedimiento técnico-administrativo de EIA en la Comunidad Valenciana, mediante el análisis de contenido de los expedientes EIA finalizados durante 2014-2015. Se examinan, entre otras cuestiones, la tipología de elementos sociales evaluados y el rigor técnico en el análisis social. Este trabajo revela importantes deficiencias metodológicas y carencias en la profundidad de la atención recibida por parte de los aspectos sociales en este tipo de procedimientos de evaluación.

Palabras clave: Evaluación de impacto ambiental, impacto social, Comunidad Valenciana, metodología, análisis de contenido

\footnotetext{
*Autor para correspondencia / Corresponding author: Guadalupe Ortiz. Universidad de Alicante. Departamento de Sociología I, Alicante, España. APD0. 9903080, Alicante, España.
}

Sugerencia de cita / Suggested citation 0rtiz, G. (2019). El tratamiento de la dimensión social en los estudios de impacto ambiental: Análisis de contenido aplicado al caso de la Comunidad Valenciana Revista Española de Sociología, 28 (1), 113-131.

(Doi: http://dx.doi.org/10.22325/fes/res.2018.59) 


\section{INTRODUCCIÓN}

La Evaluación de Impacto Ambiental (EIA) cuenta ya con treinta años de historia en nuestro país. Este instrumento, nacido en Estados Unidos a finales de la década de los sesenta, se incorpora como procedimiento administrativo en España en el año 1986, con la aprobación del Real Decreto 1302/1986. Casi desde sus inicios, esta herramienta de evaluación ha recibido críticas por parte de científicos sociales que han puesto de manifiesto las carencias que este instrumento presenta en su atención a la dimensión social, tanto en las labores de diagnóstico como en las de identificación de impactos sociales 0 establecimiento de medidas protectoras y de vigilancia. Han pasado ya veintitrés años desde que Mercedes Pardo (1994) publicara su artículo "El impacto social en las Evaluaciones de Impacto Ambiental: su conceptualización y su práctica" en la Revista Española de Investigaciones Sociológicas, en el que constataba las importantes insuficiencias de la aplicación de EIA en España a la hora de atender a los elementos sociales presentes y derivados de las intervenciones en el medio que se sometían a evaluación. A partir de su análisis de casos de ElA de embalses y carreteras, la autora confirmaba que aquellos primeros Estudios de Impacto Ambiental ${ }^{1}$ (EsIA) adolecían de un exceso de superficialidad en el tratamiento de los aspectos sociales, un mal uso de las fuentes, técnicas y datos, una evidente desconexión entre las dimensiones ambiental y social, la inexistencia de análisis interdisciplinares y la debilidad de los procesos participativos empleados en el marco de estos procedimientos.

Estas carencias no se han detectado exclusivamente en el caso español. A nivel internacional, son muchos los autores que han incidido en la necesidad de que la EIA incorpore de forma efectiva elementos de análisis social que permitan atender

1 El Estudio de Impacto Ambiental es el documento técnico que presenta el promotor del proyecto ante la administración competente para ser sometido a evaluación y que suele incorporar los siguientes apartados: a) Descripción de la acción, b) Estudio de Alternativas, c) Inventario ambiental (descripción del medio en sus distintas dimensiones), d) Identificación y Valoración de Impactos, e) Medidas protectoras o correctoras, f) Plan de vigilancia y seguimiento. a la compleja relación existente entre medio ambiente y sociedad. Recientemente, Dendena y Dorsi (2015), en un análisis global — con especial atención al caso europeo - sobre la integración de las dimensiones ambiental y social en las Evaluaciones de Impacto, señalan la falta de competencia de estos procedimientos a la hora de abordar el carácter multifacético de los impactos y reclaman una mayor atención por parte de la comunidad científica, legisladores y técnicos para poner solución a estas deficiencias. En la misma línea, Morrison-Saunders y Arts (2005: 171) apuntan que, a pesar de la creciente demanda de integración de los aspectos sociales en la EIA, "debería dedicarse más atención a los aspectos socio-económicos, tanto en las fases de pre-decisión como de seguimiento". En este sentido, tal y como expresan autores como Domínguez-Gómez (2015) o Torriti (2011), la ciencia natural y los enfoques de racionalidad instrumental están sobrerrepresentados en la EIA, observándose una clara tendencia a ignorar aquellos aspectos del proceso que le aportan los mayores niveles de complejidad e incertidumbre, esto es, los elementos socioculturales, cualitativos, de valor emocional 0 de justicia social, entre otros.

En esta misma línea, la necesidad de incorporar y hacer explícito el componente axiológico en las evaluaciones de impacto, ha sido destacado por autores como Lee et al. (1995), Hellström y Jacob (1996), Wilkins (2003), que ponen de manifiesto lo érroneo de atender únicamente a los aspectos técnicos e ignorar la carga valorativa inherente a la definición e interpretación de los impactos. Encontramos igualmente una fructífera línea de trabajo que abunda en la necesidad de incorporar estrategias de participación pública como vía para la consideración efectiva de la dimensión social en el procedimiento EIA, siendo la cuestión participativa otro de los aspectos fallidos detectados la práctica acumulada en materia de evaluación de impacto (Clark, 1994; Tilleman, 1995; Palerm, 2000; Cashmore, 2004; Hartley y Wood, 2005; 0'Faircheallaigh, 2010; Glucker et al., 2013; Salomons y Hoberg, 2014).

Este reconocimiento por parte del mundo académico de que la EIA muestra desde sus inicios importantes deficiencias en el tratamiento de las implicaciones socioculturales en la evaluación de 
proyectos, planes o programas, ha impulsado toda una línea de trabajo que ha resultado en la propuesta de un procedimiento equivalente a la EIA, pero que atiende de forma explícita a la dimensión social, esto es, la Evaluación de Impacto Social (EIS), impulsada, entre otros, por autores como F. Vanclay (2002, 2003), Burdge (2004), H. Becker (2001; Becker y Vanclay, 2003), Esteves (Esteves et al., 2012; Vanclay y Esteves (2011), o Barrow (2000). La EIS, que consta de una serie de fases similares a las que suelen articular las EIA, puede plantearse como un complemento al procedimiento habitual de EIA, pero se aplica cada vez más como instrumento de evaluación independiente para el análisis de las repercusiones sociales de un proyecto. Por otro lado, existen propuestas metodológicas que plantean de forma más clara la integración de los aspectos sociales en el procedimiento EIA tradicional, a través de los que algunos han denominado Evaluación de Impacto Integral (Rossini y Porter, 1983; Bond et al., 2001; Lee, 2006), Evaluación de Sostenibilidad (Pope et al., 2004; Bond y Morrison-Saunders, 2011) o Evaluación de Impacto Ambiental y Social (Vanclay y Bronstein, 1995; Barrow, 1997; Momtaz, 2006; Momtaz y Kabir, 2013; Dendena y Dorsi, 2015).

Esta fructífera línea de trabajo aplicado refleja la necesidad — que a su vez fundamenta el origen mismo de la Sociología Ambiental— de incorporar enfoques teórico-prácticos de naturaleza transdisciplinar a la investigación y gestión de problemas ambientales. Desde la aparición de propuestas teóricas como el "complejo ecológico" de Duncan, pasando por las reelaboraciones de Catton y Dunlap o Norgaard (Aledo y Domínguez, 2001; García, 2004) o las reflexiones en torno al propio concepto de desarrollo sostenible (López et al., 2018), ha sido constante la insistencia de la Sociología Ambiental en análisis que consideren la interdependencia de factores ambientales, demográficos, tecnológicos y socio-culturales para una correcta comprensión de la complejidad de los problemas ambientales. La puesta en evidencia de que la tradición tecnocrática y el dominio de las Ciencias Naturales habían ignorado durante largo tiempo la naturaleza antrópica de los problemas ambientales, así como las desiguales consecuencias que éstos tienen sobre los grupos sociales, son uno de los principales logros de la Sociología Ambiental (Aledo y Domínguez, 2001), y se manifiestan de forma específica en las críticas a las Evaluaciones de Impacto Ambiental.

Sin embargo, estas múltiples demandas de aumentar los niveles de integración y de aplicación de enfoques inter y transdisciplinares no han tenido por el momento una trasposición práctica en la normativa que regula los procedimientos administrativos de evaluación de proyectos con incidencia en el ámbito socio-ambiental. Así, en el caso español la EIA continúa siendo - junto a la relativamente reciente, Evaluación Ambiental Estratégica- la herramienta exigida por parte de las instituciones públicas y no existe ningún tipo de requerimiento legal que obligue a la aplicación estas otras herramientas en las que la dimensión social comparte protagonismo con la dimensión ambiental-física.

Ante esta situación, este trabajo se plantea como pregunta de investigación si, a pesar de la inexistencia de exigencias administrativas que aseguren la incorporación de procedimientos tales como EIS, Ell 0 EIAS, estas demandas planteadas desde hace años por parte del mundo académico han tenido alguna incidencia en la práctica habitual de la EIA en el caso español. Debe tenerse en cuenta que la normativa que regula la EIA, tanto a nivel europeo como a nivel nacional, ha ido incorporando progresivamente elementos de análisis social, si bien no se establece el nivel de detalle y profundidad en que éstos deben ser abordados. Centrándonos específicamente en el caso español, la reciente ley 21/2013 de evaluación ambiental, en su Artículo 2 relata los principios que fundamentan el procedimiento EIA, señalando de forma explícita a la "participación pública" como uno de esos pilares. Del mismo modo, en su Capítulo II, Artículo 35 c), relativo a los elementos que ha de incluir el Estudio de Impacto Ambiental presentado por el promotor del proyecto, se establece que este documento habrá de atender a los efectos del proyecto sobre las poblaciones humanas:

[e]valuación y, si procede, cuantificación de los efectos previsibles directos 0 indirectos, acumulativos y sinérgicos del proyecto sobre la población, la salud humana, la flora, la fauna, la biodiversidad, la geodiversidad, el suelo, el subsuelo, el aire, el 
agua, los factores climáticos, el cambio climático, el paisaje, los bienes materiales, incluido el patrimonio cultural, y la interacción entre todos los factores mencionados, durante las fases de ejecución, explotación y en su caso durante la demolición 0 abandono del proyecto.

Igualmente, el Anexo IV de dicha normativa, en el que se detalla el contenido del Estudio de Impacto Ambiental, establece que la descripción del proyecto deberá incorporar:

Un examen multi-criterio de las distintas alternativas que resulten ambientalmente más adecuadas, incluida la alternativa cero, o de no actuación, y que sean técnicamente viables, y una justificación de la solución propuesta que tendrá en cuenta diversos criterios, económico, funcional, entre los que estará el ambiental. La selección de la mejor alternativa deberá estar soportada por un análisis global multi-criterio donde se tenga en cuenta no sólo aspectos económicos sino también los de carácter social y ambiental.

Por último, cabe destacar en ese mismo anexo la aclaración que se hace en la definición de lo que el EsIA considerará como "efecto positivo" del proyecto evaluado, definiéndose en este sentido aquél que es "admitido como tal, tanto por la comunidad técnica y científica como por la población en general, en el contexto de un análisis completo de los costes y beneficios genéricos y de las externalidades de la actuación contemplada", implicando dicha definición la atención a la percepción social de los impactos positivos o beneficios del proyecto. En este punto, debe señalarse que esta particularidad solo es aplicable, según esta normativa, a los efectos positivos y no a los negativos.

A partir del contexto tanto académico como normativo que se ha expuesto, el presente trabajo persigue ofrecer una descripción y análisis detallado del modo en que todos estos elementos se incorporan efectivamente al caso español, de modo que sea posible comprobar si se han producido cambios significativos en el tratamiento de la dimensión social, tanto con motivo de los cambios normativos, como a partir de las múltiples demandas y experiencias prácticas que en este sentido se han emitido por parte de la comunidad científica en los últimos cuarenta años.

Al indagar en la existencia de aportaciones de relevancia a esta línea de investigación para el caso español, encontramos únicamente el ya citado trabajo de Mercedes Pardo (1994, 1996), así como en de Echavarren (2007), que realiza, en la línea de Pardo, una relación de los elementos sociales a los que debería atenderse en la elaboración de un Estudio de Impacto Ambiental. Este trabajo tiene un claro carácter propositivo y no cuenta con un análisis aplicado a casos concretos. También cabe señalar el trabajo de Cantó y Riera (2003) que analizan, aunque de forma superficial, el contenido socioeconómico de las EIA para el caso catalán. № se han encontrado, por tanto, análisis detallados 0 sistemáticos recientes que permitan tener una visión profunda del modo en que las EIA incorporan en la actualidad los elementos sociales en el procedimiento de evaluación.

El trabajo que aquí se presenta toma estas investigaciones como punto de partida, así como las propuestas teórico-metodológicas de la Evaluación de Impacto Social, para ofrecer un diagnóstico actualizado de la situación en la que se encuentran las Evaluaciones de Impacto Ambiental en la Comunidad Valenciana. De este modo, se persigue averiguar si las demandas y propuestas que se han realizado desde la academia en los últimos veintidós años han fructificado en una mejor atención a los elementos sociales en los Estudios de Impacto Ambiental, en comparación con las deficiencias detectadas por Pardo a principios de los años 90. Para ello, se ha llevado a cabo un exhaustivo y sistemático análisis de contenido de 51 Estudios de Impacto Ambiental pertenecientes a los expedientes de EIA tramitados desde el 1 de enero de 2014 hasta junio de 2015 en la Generalitat Valenciana. Este análisis se ha centrado aquellos bloques de contenido de los Estudios de Impacto Ambiental en los que es posible obtener una visión más clara del modo en que el estudio de los elementos sociales se incorpora a los Estudios de Impacto Ambiental, esto es, el apartado de Medio Socio-Demográfico incluido en la fase de Inventario Ambiental y el apartado de Identificación y Evaluación de Impactos. 


\section{DISEÑO METODOLÓGICO Y ANALÍTICO}

Tal y como se ha indicado, este trabajo se ha basado en el análisis de contenido los documentos correspondientes a los Estudios de Impacto Ambiental de 51 expedientes tramitados durante el año 2014 y la primera mitad del año 2015 (cuando da comienzo el presente proyecto). Para la configuración de la muestra de documentos se accedió a la base de datos facilitada por el Servicio de Evaluación Ambiental de la Generalitat Valenciana, que registraba un total de 123 expedientes tramitados en las fechas indicadas. A continuación, se hizo un primer cribado de los expedientes, seleccionando aquellos que contaran con copia digitalizada del Estudio de Impacto Ambiental y aquellos para los que hubiera finalizado el procedimiento administrativo, es decir, contaran con declaración de impacto ambiental, identificándose un total de 56 expedientes que reunían ambos requisitos. A continuación, se procedió a la homogeneización de formatos de los documentos y a su preparación para el análisis mediante Atlas.ti, sometiendo los archivos a un proceso de Optical Character Recognition (OCR) en los casos en los que resultó necesario. En cinco casos, este tratamiento de los documentos dio lugar a errores en el reconocimiento del texto y fue necesario eliminar dichos expedientes de la muestra. De este modo, la muestra final estaba conformada por un total de 51 documentos de Estudio de Impacto Ambiental. La siguiente tabla muestra la tipología de proyectos evaluados incluidos en dicha muestra:

Tabla 1. Tipología de proyectos evaluados incluidos en la muestra

\begin{tabular}{lc}
\hline TIPO DE PROYECTO EVALUADO & Recuento \\
\hline Gestión y almacenamiento de residuos & 30 \\
\hline Explotación ganadera & 11 \\
\hline Carreteras & 3 \\
\hline Gestión de recursos hídricos & 2 \\
\hline Líneas y estaciones eléctricas & 3 \\
\hline Infraestructuras aeronáuticas & 1 \\
\hline Gasoductos & 1 \\
\hline Total & 51 \\
\hline
\end{tabular}

Según la tipología de análisis establecida por Conde (2009) en su revisión del amplio espectro de procesos analíticos en el ámbito del análisis de discurso sociológico, y que oscilan desde el análisis de contenido clásico (Berelson, 1952; Krippendorf, 1980) a la socio-hermenéutica (Alonso, 1998, 2013), el procedimiento aquí empleado puede definirse como un análisis de contenido que combina el análisis temático y por categorías. Este análisis estuvo apoyado en el uso del software informático Atlas.ti. En este sentido, el análisis se fundamentó en una identificación inicial de las dimensiones y códigos de análisis para el estudio pormenorizado de los documentos. En este artículo, nos vamos a centrar en el análisis de cuatro dimensiones principales: la tipología de elementos sociales tratados en el apartado socioeconómico que habitualmente se incluye en la fase de Inventario Ambiental de un EsIA; la tipología de impactos y procesos de cambio social tratados en el apartado de Identificación y Evaluación de Impactos; las herramientas de análisis social empleadas en los dos apartados anteriores, así como criterios de evaluación del uso de las mismas; y la naturaleza disciplinar de los equipos de trabajo. A partir de estas dimensiones es posible observar de forma clara el contenido y características de las referencias a aspectos sociales existentes en los documentos técnicos de los EsIA, bien en tanto que elementos de diagnóstico inicial (Inventario), bien en relación a la identificación de impactos sociales, 0 bien a partir del carácter multidisciplinar de los equipos de trabajo que desarrollan el EsIA.

Para el análisis de cada una de estas dimensiones se identificaron una serie de códigos específicos, fundamentados cada uno de ellos en una revisión previa de la literatura especializada. En relación al análisis de los apartados socioeconómicos, se han tomado como referencia los trabajos de Pardo y Echevarren, cuyas propuestas han sido combinadas y ampliadas dando lugar a una batería de códigos que permitiera identificar el tipo de elementos sociales a los que suele atenderse en la elaboración de este apartado de diagnóstico por parte de los equipos técnicos que desarrollan los EsIA.

A continuación, la Tabla 2 ofrece el listado de códigos empleados para el análisis de esta dimensión, así como la explicación del significado de cada uno de ellos. 
El tratamiento de la dimensión social en los estudios de impacto ambiental: Análisis de contenido aplicado al caso...

Tabla 2. Códigos empleados en el análisis de la tipología de elementos sociales referenciados en el apartado de Medio Socioeconómico de los EsIA

\begin{tabular}{|c|c|}
\hline $\begin{array}{l}\text { ELEMENTO: } \\
\text { actividad: construcción }\end{array}$ & $\begin{array}{l}\text { Referencias relativas a la actividad urbanística, constructiva, sector de la vivienda, } \\
\text { infraestructuras, etc. }\end{array}$ \\
\hline $\begin{array}{l}\text { ELEMENTO: } \\
\text { actividad: industria }\end{array}$ & Referencias relativas a la actividad económica industrial, manufacturera, etc. \\
\hline $\begin{array}{l}\text { ELEMENTO: } \\
\text { actividad: sector primario }\end{array}$ & $\begin{array}{l}\text { Referencias relativas a la actividad económica agrícola, ganadera, pesquera, silvicultora, } \\
\text { etc. }\end{array}$ \\
\hline ELEMENTO: actividad: servicios & Referencias relativas a la actividad económica terciaria, sector servicios, comercio, etc. \\
\hline ELEMENTO: conflicto & $\begin{array}{l}\text { Referencias relativas a la existencia de conflictos sociales, movilizaciones, actos de } \\
\text { protesta, debate intergrupal, etc. }\end{array}$ \\
\hline ELEMENTO: cultura & $\begin{array}{l}\text { Referencias relativas a los elementos culturales propios de las comunidades estudiadas, } \\
\text { sus costumbres, tradiciones, patrimonio, folclore, lengua, identidad, elementos simbólicos } \\
\text { y estéticos, etc. }\end{array}$ \\
\hline ELEMENTO: demografía & $\begin{array}{l}\text { Referencias relativas a demografía, movimientos poblacionales, estructura poblacional, } \\
\text { indicadores demográficos, estructura familiar, etc. }\end{array}$ \\
\hline ELEMENTO: desigualdad & $\begin{array}{l}\text { Referencias relativas a situaciones o diagnósticos de desigualdad social, situaciones de } \\
\text { vulnerabilidad socio-ambiental, especial atención a grupos de riesgo, etc. }\end{array}$ \\
\hline ELEMENTO: economía & Referencias a la actividad económica general, relaciones de consumo, propiedad, etc. \\
\hline ELEMENTO: empleo & $\begin{array}{l}\text { Referencias relativas al ámbito laboral, niveles y características del empleo, indicadores } \\
\text { de desempleo, actividad, empresa, etc. }\end{array}$ \\
\hline ELEMENTO: entorno & $\begin{array}{l}\text { Referencias relativas a las condiciones ambientales con afectación directa sobre las } \\
\text { poblaciones en materia de ruido, olores, vibraciones, iluminación, calidad atmosférica, etc. }\end{array}$ \\
\hline $\begin{array}{l}\text { ELEMENTO: } \\
\text { entorno: paisaje }\end{array}$ & $\begin{array}{l}\text { Referencias relativas al paisaje, calidad paisajística, percepción del paisaje, } \\
\text { características estéticas del entorno }\end{array}$ \\
\hline ELEMENTO: género & Referencias relativas a cuestiones de género, desigualdad entre hombres y mujeres, etc. \\
\hline ELEMENTO: infraestructuras & Referencias a infraestructuras de uso público (ej. carreteras) \\
\hline ELEMENT0: legislación & Referencias relativas al ámbito legislativo y jurídico, normativas y regulaciones, etc. \\
\hline ELEMENTO: ocio & $\begin{array}{l}\text { Referencias relativas al ocio, actividades recreativas y lúdicas, turismo, tiempo libre, } \\
\text { aficiones, deporte, etc. }\end{array}$ \\
\hline ELEMENTO: otros & Otras referencias a elementos sociales no incluidas en el resto de categorías. \\
\hline ELEMENTO: percepción & $\begin{array}{l}\text { Referencias relativas a la percepción social, estudios de opinión, niveles de satisfacción } 0 \\
\text { preocupación, atribución de significados, etc. }\end{array}$ \\
\hline ELEMENTO: poder & $\begin{array}{l}\text { Referencias relativas a cuestiones de poder, capacidad de influencia en los procesos de } \\
\text { toma de decisiones de los grupos sociales, etc. }\end{array}$ \\
\hline $\begin{array}{l}\text { ELEMENTO: } \\
\text { política, democracia }\end{array}$ & $\begin{array}{l}\text { Referencias relativas a la actividad política y democrática, participación pública, } \\
\text { asociacionismo y sindicalismo, empoderamiento, autogestión, etc. }\end{array}$ \\
\hline $\begin{array}{l}\text { ELEMENTO: } \\
\text { salud, calidad vida }\end{array}$ & $\begin{array}{l}\text { Referencias relativas a cuestiones de salud e insalubridad, enfermedades, condiciones } \\
\text { sanitarias y médicas, nutricionales, psicosociales, (estrés, depresión, etc.), alergias, } \\
\text { afecciones respiratorias, etc. }\end{array}$ \\
\hline ELEMENTO: seguridad & $\begin{array}{l}\text { Referencias relativas a la seguridad de las poblaciones, niveles de criminalidad y } \\
\text { delincuencia, sistemas de vigilancia, etc. }\end{array}$ \\
\hline ELEMENTO: transporte & $\begin{array}{l}\text { Referencias relativas a la movilidad de las personas, accesibilidad, transporte, tráfico, } \\
\text { vías de comunicación, etc. }\end{array}$ \\
\hline
\end{tabular}


El tratamiento de la dimensión social en los estudios de impacto ambiental: Análisis de contenido aplicado al caso...

En el caso del análisis de los apartados de Identificación y Evaluación de Impactos, se ha tomado como referencia para el análisis el trabajo de conceptualización realizado por Vanclay (2002) y que ha permitido la elaboración de un listado de códigos que reflejan la distinción que este autor realiza entre los conceptos de impacto social y proceso de cambio social. Para Vanclay (2002: 191), los impactos sociales se refieren a "impactos efectivamente experimentados por las personas (a nivel individual 0 agregado) en un sentido corpóreo (físico) o cognitivo (perceptual)"2, mientras que los procesos de cambio social tienen un carácter más sistémico y/o estructural, pudiendo tales procesos dar lugar a impactos en última instancia. Como señala el autor "el cre- cimiento poblacional o la presencia de trabajadores de la construcción" no son en sí mismos impactos sino procesos de cambio que dan lugar a impactos. Ambos elementos son objeto de análisis por parte de las Evaluaciones de Impacto Social y han sido tomados como referencia para el análisis que aquí se presenta. La siguiente tabla ofrece la relación de códigos empleada, así como una breve descripción del significado de los mismos. No se han incluido las definiciones detalladas de cada código ya que éstas aparecen pormenorizadas en el artículo de Vanclay tomado como referencia. Es por ello que, para una interpretación completa del significado de los códigos, nos remitimos en todo momento a la obra de referencia indicada.

Tabla 3. Códigos empleados en el análisis de los procesos de cambio e impactos sociales en el apartado de Identificación y Valoración de impactos del Estudio de Impacto Ambiental

\begin{tabular}{|c|c|}
\hline $\begin{array}{l}\text { PROCESOCAMBIO: } \\
\text { demográfico }\end{array}$ & $\begin{array}{l}\text { Procesos demográficos, transformaciones en el volumen y la composición de la población. } \\
\text { Procesos de inmigración, emigración, presencia de recién llegados, presencia (temporal) } \\
\text { de trabajadores de la construcción, de residentes estacionales, turistas o excursionistas, } \\
\text { re-asentamiento, desplazamiento y expropiación, éxodo rural y éxodo urbano }\end{array}$ \\
\hline $\begin{array}{l}\text { PROCESOCAMBIO: } \\
\text { económico }\end{array}$ & $\begin{array}{l}\text { Procesos económicos relacionados con la forma en que las personas se ganan la vida } \\
\text { y las actividades económicas. Conversión y diversificación de actividades económicas, } \\
\text { empobrecimiento, inflación, fluctuación de cambio de moneda (devaluación), } \\
\text { concentración de la actividad económica y globalización económica (conversión a la } \\
\text { producción orientada al mercado mundial). }\end{array}$ \\
\hline $\begin{array}{l}\text { PROCESOCAMBIO: } \\
\text { empoderamiento }\end{array}$ & $\begin{array}{l}\text { Procesos emancipatorios y de empoderamiento, de creciente influencia en los procesos } \\
\text { de toma de decisiones. Democratización, marginación y exclusión y capacidad de } \\
\text { construir, aumento de conocimientos y habilidades y creación de redes en la población } \\
\text { local. }\end{array}$ \\
\hline $\begin{array}{l}\text { PROCESOCAMBIO: } \\
\text { geográfico }\end{array}$ & $\begin{array}{l}\text { Procesos geográficos y cambios en los patrones de uso de la tierra. Conversión y } \\
\text { diversificación de los usos de la tierra, expansión urbanística (en zonas rurales o pre- } \\
\text { urbanas), urbanización, éxodo rural así como el aumento de población en los núcleos de las } \\
\text { ciudades, gentrificación, mejoras en el transporte y la accesibilidad rural, fractura o división } \\
\text { física (fragmentación de las comunidades debido a proyectos de grandes infraestructuras). }\end{array}$ \\
\hline $\begin{array}{l}\text { PROCESOCAMBIO: } \\
\text { institucional, legal }\end{array}$ & $\begin{array}{l}\text { Procesos institucionales y legales, relativos a la eficiencia y efectividad de las } \\
\text { estructuras institucionales incluyendo organizaciones gubernamentales y no } \\
\text { gubernamentales. Globalización institucional y centralización (incorporación de lo local } \\
\text { en lo global, pérdida de influencia en la decisión a nivel local), descentralización y } \\
\text { privatización }\end{array}$ \\
\hline $\begin{array}{l}\text { PROCESOCAMBIO: } \\
\text { sociocultural }\end{array}$ & $\begin{array}{l}\text { Procesos socioculturales. Globalización sociocultural, segregación, creación de } \\
\text { diferencias sociales, desintegración social, pérdida de capital social (redes sociales, } \\
\text { culturales, parentesco, prácticas culturales, etc.) y diferencias culturales (valores, } \\
\text { tradiciones, rituales, lenguaje, etc.). }\end{array}$ \\
\hline
\end{tabular}

2 Traducción propia. 
El tratamiento de la dimensión social en los estudios de impacto ambiental: Análisis de contenido aplicado al caso...

PROCESOCAMBI0: otros Otros procesos de cambio social no incluidos en las categorías anteriores.

IMPACT0: Calidad ambiental percibida (polvo, ruido, riesgo, olor, vibración, contaminación lumínica, calidad vida, entorno seguridad, aglomeración, presencia de extraños, traslados, etc.), calidad del medio real, interrupción de la rutina, ocio y tiempo libre, valor estético (impactos visuales, panorámica, vistas, etc.), armonía ambiental, percepción de la calidad de las viviendas, calidad de las viviendas real, percepción de la calidad social de la vivienda (hogar), disponibilidad de las instalaciones de la vivienda, infraestructuras (agua, aguas residuales, tierra, carreteras, etc.), infraestructuras sociales (educación, policía, bibliotecas, asistencia social, etc.), percepción de la seguridad física y miedo al crimen, seguridad física y exposición al peligro real, crimen y violencia.

\begin{tabular}{ll}
\hline IMPACT0: & Cambios en los valores culturales, pérdida o violación de lugares sagrados, tabús, etc., \\
cultura & integridad cultural, sentimiento de marginación hacia la cultura (exclusión social de \\
& ciertos grupos, profanación de la cultura) explotación comercial o mercantilización del \\
& patrimonio cultural y su consiguiente pérdida de significado, pérdida del lenguaje local o \\
& dialecto y pérdida de patrimonio natural y cultural. \\
\hline IMPACT0: & Carga de trabajo (por supervivencia o por calidad de vida), calidad de vida y riqueza \\
economía, recursos & (hogar y servicios), acceso a bienes y servicios públicos, acceso a la administración \\
& y/u otros servicios sociales, prosperidad económica y resiliencia, renta (efectivo o en \\
& especie), valor de la propiedad, estatus o prestigio profesional y tipo de empleo, nivel de \\
& desempleo de la comunidad, pérdida de oportunidades de empleo, costes de reposición \\
& de servicios ambientales, dependencia económica o vulnerabilidad, trastorno de la \\
& economía local y deuda nacional.
\end{tabular}

IMPACT0: $\quad$ Alteración de la estructura familiar (estabilidad familiar, divorcios, número de hijos familia, comunidad en casa, presencia de familias extensas, etc.), cambios en las relaciones sexuales, cargas familiares por ancianos y/o antepasados, violencia familiar, alteración de las redes sociales (impactos en la interacción social entre los miembros de la comunidad), cambios en la estructura demográfica de la comunidad, identidad y conexión, cohesión percibida y real, diferencias sociales y tensión social y violencia.

IMPACTO: $\quad$ Carga y viabilidad de la administración 0 agencias formales, carga y viabilidad de institucional, legal, $\quad$ organismos no gubernamentales y agencias informales (incluyendo organizaciones político, igualdad comunitarias), integridad de agencias gubernamentales y no gubernamentales, pérdida de tenencia o derechos legales, pérdida de subsidiariedad, violación de los derechos humanos, participación en el proceso de toma de decisiones, acceso a procedimientos legales y asesoramiento jurídico y equidad de los impactos.

IMPACTO: Integridad física de la mujer (capacidad de tomar decisiones sobre su propio cuerpo, relaciones género salud, actividad sexual, fertilidad, maternidad, crianza de hijos y tenencia de recursos necesarios para tomar esas decisiones libres de coerción, violencia o discriminación), autonomía personal de la mujer, división sexual del trabajo, división sexual de las tareas del hogar, división sexual de las tareas reproductivas del hogar, acceso a recursos y servicios (tierra, agua, capital, educación, empleo, salud, etc.), igualdad educacional y políticas de emancipación de la mujer.

IMPACT0: $\quad$ Fallecimiento o pérdida personal, pérdida de población o capital social, nutrición, salud y salud, bienestar fertilidad real (capacidad de concebir), salud y fertilidad observada, salud mental, cambio en las aspiraciones futuras de los hijos, autonomía (cambios en la independencia del individuo), desviación social, incertidumbre, sentimientos positivos o negativos de la intervención social, molestias (debido a la interrupción de la vida cotidiana), insatisfacción y sentimiento de traición (ausencia de los beneficios prometidos) e indignación moral (provocada por la violación de creencias morales o religiosas).

IMPACTO: otros Otros impactos no incluidos en las categorías anteriores. 
Por otro lado, se ha creado una batería compuesta por un total de 13 códigos destinados a la identificación de los procedimientos técnicos empleados para la recogida de información, tanto en la fase de inventario socio-económico como en la de identificación y valoración de impactos. Con ello se perseguía comprender los elementos metodológicos que fundamentan la identificación de procesos/impactos sociales, así como el uso que de estas herramientas se hace por parte de los equipos técnicos. Los códigos empleados para ello son:

-Encuesta

-Entrevista cualitativa

- Grupo de discusión

-Talleres

-Alegaciones

— Recogida de datos secundarios

—Documentación

- Consultas/peticiones informales

- Observación directa

-Matriz de impactos

-Mediciones técnicas (ej. mediciones acústicas)

-Negociaciones entre partes

- Otras técnicas

Para el análisis de los procedimientos metodológicos empleados, se atendió durante el proceso de codificación a cuestiones tales como:

- Calidad en el uso de la técnica, que perseguía valorar la calidad técnica en el empleo de herramientas de investigación social y detectar errores en su uso.

- Calidad en el diseño muestral, aplicable a aquellos casos en los que la selección de muestras era requerido por la técnica (ej. encuesta).

-Nivel de transparencia o claridad en la exposición del procedimiento metodológico y en las fuentes de datos.

-Nivel de adecuación y relevancia de las técnicas y datos utilizados respecto de los objetivos buscados.

-Nivel de actualización de los datos.

Finalmente, se analizaron los distintos documentos técnicos a partir de las características de los equipos redactores, atendiendo al número de miembros que los conforman, así como a las áreas de especialización de cada uno de los miembros del equipo. Con ello, se perseguía obtener información acerca del carácter multidisciplinar del equipo, la capacidad de atender al carácter multifacético de los impactos sobre el entorno y, específicamente, a la presencia de científicos sociales en estos grupos de trabajo.

\section{RESULTADOS}

El primer objeto de análisis de esta investigación ha sido el contenido de los apartados dedicados al estudio del contexto socioeconómico incluidos en la fase de Inventario Ambiental de los EsIA. Se han identificado 41 documentos en los que existía un apartado dedicado a esta cuestión, existiendo 10 documentos en los que no se ha realizado ningún tipo de estudio socioeconómico previo a la fase de identificación de impactos. Estos documentos que no ofrecen apartado socioeconómico se refieren a EsIA para instalaciones ganaderas (2) y avícolas (4), así como de instalaciones de almacenamiento de chatarra (3) y almacenamiento de residuos peligrosos y no peligrosos (1).

En los 41 documentos en los que sí que aparece un apartado de estudio socioeconómico, se ha analizado la tipología de elementos sociales a los que se atiende en cada caso. La Tabla 4 muestra el total de citas codificadas en relación al tipo de elemento social tratado en los 41 apartados de análisis socioeconómico. Se observa cómo el foco de atención principal se centra, con 62 citas, en la descripción de las características demográficas del municipio en el que se pretende desarrollar el proyecto evaluado. En segundo lugar, aparecen los análisis relativos al estudio de los sectores económicos presentes en la zona de afectación, con especial atención al sector primario, seguido por el terciario, secundario y de la construcción y otras cuestiones de carácter económico (niveles de ocupación, contratación, paro registrado, etc.). Se encuentran también, aunque en menor medida, referencias en estos apartados a infraestructuras de uso público, así como a elementos de tipo cultural (referidos fundamentalmente a la descripción de elementos 
El tratamiento de la dimensión social en los estudios de impacto ambiental: Análisis de contenido aplicado al caso...

patrimoniales arqueológicos 0 arquitectónicos). En ningún caso se dedica atención a áreas de estudio clásicas de la disciplina sociológica, tales como relaciones de conflicto, desigualdad, género 0 análisis de percepción u opinión.

Tabla 4. Recuento de citas según código de Elemento Social en fase de Inventario Socioeconómico

\begin{tabular}{lc}
\hline CóDIGOS DE ELEMENTO SOCIAL & Recuento de citas $^{3}$ \\
\hline ELEMENTO: demografía & 62 \\
\hline ELEMENTO: actividad: sector primario & 57 \\
\hline ELEMENTO: actividad: servicios & 51 \\
\hline ELEMENTO: actividad: industria & 48 \\
\hline ELEMENTO: actividad: construcción & 40 \\
\hline ELEMENTO: empleo & 31 \\
\hline ELEMENTO: economía & 23 \\
\hline ELEMENTO: infraestructuras & 15 \\
\hline ELEMENTO: cultura & 10 \\
\hline ELEMENTO: transporte & 8 \\
\hline ELEMENTO: economía & 6 \\
\hline ELEMENTO: entorno: paisaje & 5 \\
\hline ELEMENTO: entorno & 4 \\
\hline ELEMENTO: ocio & 3 \\
\hline ELEMENTO: política, democracia & 3 \\
\hline ELEMENTO: legislación & 2 \\
\hline ELEMENTO: salud, calidad vida & 2 \\
\hline ELEMENTO: seguridad & 2 \\
\hline ELEMENTO: conflicto & 0 \\
\hline ELEMENTO: desigualdad & 0 \\
\hline ELEMENTO: género & 0 \\
\hline ELEMENTO: percepción & 0 \\
\hline ELEMENTO: poder & \\
\hline
\end{tabular}

3 Se ha optado por la exposición de los resultados en forma de frecuencia absoluta y no relativa debido a que una misma cita puede contener más de un código, por lo que tomar el sumatorio total de número de citas como número de referencia para el cálculo de porcentajes podría ofrecer una interpretación engañosa de la distribución de códigos por citas.
La ausencia de análisis que requieran de un estudio más profundo de la realidad social se explica, en cierta medida, atendiendo a las herramientas de recogida de información que se emplean para elaborar estos apartados de los documentos EsIA (Tabla 5). El empleo de datos estadísticos secundarios y documentación constituyen la estrategia empleada casi en exclusiva para realizar la descripción del contexto socioeconómico del área de afectación del proyecto. En ningún caso se realizan labores de entrevista u observación que requieran del contacto directo con la realidad social objeto de estudio. En 48 ocasiones, se ofrece información para la cual no se especifica la herramienta de recogida de datos empleada, lo cual es indicador de una importante falta de transparencia metodológica que podría poner en cuestión el contenido de estos informes.

Tabla 5 . Recuento de citas de Herramientas de recogida de información empleadas en la Fase de Inventario Socioeconómico

\begin{tabular}{lc}
\hline & Recuento \\
\hline HERRAM: datos estad. secundarios & 91 \\
\hline HERRAM: Sin especificar & 48 \\
\hline HERRAM: documentación & 39 \\
\hline HERRAM: mediciones téc. & 1 \\
\hline
\end{tabular}

Atendiendo al tipo de información recabada a través de estas herramientas, se observa un claro patrón que se repite en la mayoría de los documentos independientemente de la temática 0 tipo de proyecto evaluado. En la práctica mayoría de los casos, el estudio socioeconómico se reduce a la exposición de datos demográficos generales, basados en una descripción superficial de la estructura poblacional y los datos de los padrones municipales de habitantes de la localidad, así como la exposición de una serie de indicadores pre-elaborados (en su mayoría, aquellos contenidos en las fichas municipales disponibles en la página web del Instituto Valenciano de Estadística). Esta atención a la descripción demográfica a escala municipal, si bien podría ser útil en algunos casos para con- 
seguir una visión general de las características y dinámicas poblacionales del municipio en que se desarrolla el proyecto, puede resultar en otros muchos casos irrelevante. Una atención más focalizada en el área específica de afectación del proyecto, las características de la población directamente afectada y de los asentamientos humanos colindantes resultaría de mayor utilidad para los fines de esta fase del estudio, si bien requeriría de trabajo de campo y contacto directo con el contexto social local, cuestión que parece completamente ausente de las dinámicas de trabajo actuales que sustentan los EsIA.

Del mismo modo, el análisis de la estructura económica y empresarial —que comparte protagonismo con el análisis de datos demográficosmuestra las mismas carencias que la cuestión demográfica. La exposición de datos de actividad según sectores suele reproducir las fichas estandarizadas provistas por el Instituto Valenciano de Estadística, sin guardar, necesariamente, especial relación con el tipo de proyecto estudiado. Ello deriva en una exposición de datos que resultan con frecuencia poco útiles a los propósitos del estudio de impactos. A ello se añade que, en muchas ocasiones, se ha detectado la exposición de datos poco actualizados y que, por lo tanto, no aseguran reflejar adecuadamente las características sociales de la población estudiada en el momento del diseño del proyecto.

En relación al análisis de contenido desarrollado para el apartado de Identificación y VaIoración de Impactos, se reproducen algunas de las deficiencias anteriormente apuntadas si bien se detecta una mayor diversidad de enfoques y temáticas sociales analizadas. En relación a la distinción realizada por Vanclay entre procesos de cambio e impactos sociales, la atención se focaliza de forma clara en la segunda de las dimensiones de análisis. La atención elementos de carácter sistémico y/0 estructural que pudieran, en última instancia, dar lugar a impactos sociales, es muy limitada y se reduce a la referencia a cuestiones tales como:

-El aumento de "Ios niveles de sostenibilidad", "desarrollo de la economía local", etc.
-Cambios en procesos migratorios y estructura demográfica.

- Mejora en los procesos productivos, diversificación empresarial, cambios en la estructura productiva, efectos sobre la competencia.

- Cambios en los sistemas de movilidad y conexiones, alteraciones en los flujos de tráfico.

Tabla 6. Recuento de citas según códigos de Procesos de Cambio Social e Impactos Sociales en la fase de Identificación y Valoración de Impactos

\begin{tabular}{lc}
\hline & Recuento de citas \\
\hline PROCESOCAMBI0: demográfico & 5 \\
\hline PROCESOCAMBI0: económico & 10 \\
\hline PROCESOCAMBI0: geográfico & 3 \\
\hline PROCESOCAMBI0: socioculturales & 2 \\
\hline IMPACTO: calidad vida, entorno & 285 \\
\hline IMPACT0: cultura & 2 \\
\hline IMPACTO: economía, recursos & 123 \\
\hline IMPACTO: salud, bienestar & 11 \\
\hline
\end{tabular}

En la mayoría de los casos, estas referencias muestran una ausencia de profundización 0 explicación detallada y cuentan con una fundamentación empírica dudosa. Por ejemplo, uno de los EsIA analizados, dedicado a la evaluación de un proyecto de acondicionamiento y refuerzo del firme de una carretera en un tramo de 10'2 km, identifica, entre otras cuestiones, como impactos positivos del proyecto "cambios en los procesos migratorios" y "efectos sobre la población activa de la zona". Ninguna precisión, explicación o justificación acompaña a estas dos afirmaciones, sin quedar claro en qué modo se transformarán los procesos migratorios por el acondicionamiento de un tramo de carretera convencional, ni en qué modo ello podría suponer impactos efectivos en la zona, ni en qué forma se verá afectada la población activa del área de afectación. Si el objetivo era afirmar que la ejecución del proyecto supondrá la incorporación como mano de obra de población desempleada (afectando así a miembros de la "población activa"), sería necesario que se justificara y se reflejara 
adecuadamente en este punto, dado que nada es posible inferir a este respecto a partir de la información contenida en el documento. De cualquier modo, parece que el uso del término "población activa" no es el más adecuado y tal vez se haya confundido con el de "población ocupada". Otro proyecto, dedicado a la evaluación de una instalación de explotación avícola, identifica efectos en la "estructura agraria y socioeconómica" — si bien lo justifica a través del impacto directo que tendrá sobre el promotor del proyecto-y arguye que esta instalación "generará y permitirá mantener dos puestos de trabajo y afianzará la continuación de la vida del promotor en el municipio de (nombre del municipio), reduciendo la despoblación". Otro ejemplo de identificación de procesos de cambio social es el del proyecto de una instalación ganadera en el que se indica que éste contribuirá a una "cohesión social y territorial, etc.".

Este tipo de afirmaciones poco fundamentadas se han observado con gran frecuencia a lo largo del análisis de contenido, especialmente en lo referido a la justificación de impactos positivos sobre el empleo. Así lo refleja, como se verá más adelante en la Tabla 7 , la ausencia de información metodológica que permita valorar la validez de las afirmaciones que se realizan en esta etapa de identificación de impactos.

También se han detectado casos en los que se identifican impactos derivados de proyectos distintos del evaluado. Por ejemplo, en el caso de un EsIA para la construcción de una variante, se indica que:

La desaparición del tráfico por el centro del núcleo urbano favorecerá la aparición de instalaciones hosteleras, sobre todo bares, que aprovechan las dimensiones de la antigua carretera para implantar terrazas. Para que se produzca este efecto será necesario transformar la carretera en calle y potenciar la zona como área de descanso y avituallamiento fuera de la carretera. Así pues, con medidas potenciadoras el impacto sería positivo y severo.

Es decir, se incluye como impacto positivo propio de este proyecto uno que solo ocurrirá si tiene lugar un hipotético proyecto todavía no iniciado.

Otro proyecto de instalación de un centro de transferencia de residuos, incorpora a su identifi- cación de impactos "factores científico-educativos o formativos y educativos" y acompaña esta identificación — sin más especificaciones- de un conjunto de puntuaciones numéricas que se sintetizan en una valoración de 28 puntos en importancia para impacto positivo. Algo similar ocurre en un proyecto para un almacén de chatarra en el que se incluye como impacto positivo "Impacto positivo en la concienciación de la población de la importancia de gestión de los residuos, ya no como una obligación sino como un deber social". Este tipo de valoraciones con absoluta falta de fundamentación y sin acompañamiento alguno de evidencias que los demuestren son habituales en los informes analizados.

En todos los casos, la atención a procesos de cambio social en este apartado de impactos, se ha producido asociada a la identificación de impactos positivos. Parece que el recurso a este tipo de cuestiones se emplea como excusa para favorecer una visión positiva de la actividad evaluada. Si recordamos, además, la exigencia normativa según la cual los impactos positivos han de definirse como tales "tanto por la comunidad técnica y científica como por la población en general", esta ausencia de fundamentación resulta más alarmante. En ningún caso de los 51 documentos analizados se ha comprobado que tal requerimiento se cumpla.

En relación al análisis de los impactos identificados en Ios EsIA, —entendiendo el concepto de impacto social en los términos expresados en la Tabla 3-, se observa una clara preeminencia de los impactos relativos a la calidad de vida y el entorno humano, en su mayoría en lo relativo a "cambios en el entorno real", y no en relación a cambios en el entorno 0 calidad percibida por los afectados últimos de dichos impactos y sin que exista referencia expresa al impacto directo sobre las personas. En este sentido si, por ejemplo, se alude al "riesgo de inundaciones" como impacto, no se determina a quiénes afectarían dichas inundaciones, en caso de que afectaran a alguna población humana. Del mismo modo, son muy frecuentes las referencias al aumento de ruidos pero no tanto la referencia explícita a las poblaciones humanas afectadas por ellos. Dado que interpretamos que, con gran probabilidad, la identificación del incremento de ruidos se realiza en tanto 
que éste es percibido por los humanos ${ }^{4}$, se han codificado estos casos con el código de impacto sobre la calidad de vida y el entorno. Es decir, durante el proceso de codificación se han identificado como impactos sociales aquellos que, a criterio del equipo de esta investigación, podrían haberse incluido como tales en el EsIA por su repercusión sobre las poblaciones humanas, si bien es frecuente que no se refleje explicitamente tal impacto sobre los individuos en los informes. Lo mismo ocurre con la identificación de impactos sobre la calidad atmosférica, los olores, el impacto estético sobre el paisaje, etc.

De este análisis del código "IMPACTO: calidad vida,entorno" (ver Tabla 3) destaca también que, en las escasas ocasiones en que alude al elemento perceptivo de este tipo de impactos, la conclusión del equipo redactor del EsIA no suele estar fundamentada en un contacto directo con las poblaciones que habrían de percibir dicho impacto. Es decir, si se alude a la percepción del paisaje, ésta está habitualmente fundamentada en el criterio del técnico y no en el criterio de las poblaciones que habitualmente disfrutan de dicho paisaje. En el caso de la percepción de olores, por ejemplo, tampoco se realizan estudios directos con las poblaciones afectadas, estableciéndose conclusiones poco fiables. Esta ausencia de contacto directo con las poblaciones a las que se hace referencia en los EsIA resulta especialmente llamativa cuando se hace alusión directa a la aceptación social del proyecto. Esto se ilustra de forma clara en la siguiente cita:

En cuanto a la aceptación social del proyecto, en general, para las líneas eléctricas y, dado que el suministro de energía es un bien imprescindible y necesario para la población actual, el rechazo a estas infraestructuras se ha ido atenuando notablemente.

De ello se infiere que el proyecto evaluado cuenta con aceptación social. Lo mismo ocurre en el caso de una instalación de gestión de chatarra:

4 En algunas ocasiones, la alusión a la calidad acústica podría estar referida a su impacto sobre los animales, especialmente en el caso de infraestructuras ganaderas 0 avícolas.
"En cuanto a la aceptación social, consideramos que se trata de una actividad bien aceptada, debido a que puede suponer un beneficio tanto desde el punto de vista laboral (generación de puestos de trabajos), como desde el punto de vista medioambiental, por la correcta gestión de residuos que supone la actividad empresarial".

Este tipo de asunciones y generalizaciones poco justificadas, se han detectado con cierta frecuencia en los informes analizados, especialmente para descartar la existencia de un impacto negativo 0 para fundamentar un supuesto impacto positivo. En otros casos, se equipara la ausencia de impacto negativo con un impacto positivo, como ocurre en este EsIA para la construcción de un helipuerto: "se contemplan las molestias producidas en la población por el incremento del nivel de ruido, en este caso se trata de un efecto negativo, pero debido a la lejanía de núcleos poblacionales, se puede considerar positivo en este caso".

Como muestra la Tabla 6, el segundo grupo de impactos en términos de frecuencia es el relativo a los impactos económicos y sobre los recursos materiales. Bajo el código "IMPACT0: economía, recursos" (ver Tabla 3) se han identificado tres tipos de impactos: los relativos a la creación de puestos de trabajo (si bien, en numerosas ocasiones no se contabiliza el número de puestos que el proyecto creará y en muy pocas se especifica el tipo de contratación 0 condiciones laborales del puesto); los relativos a la expropiación de propiedades e indemnizaciones económicas (sobre las que no se aportan detalles sobre las condiciones específicas en que se producirán); el impacto sobre la actividad productiva de individuos afectados (ej. por afectación a campos de cultivo, mejora en el abastecimiento recursos hídricos para riego, etc.) 0 del promotor y otras empresas relacionadas con él (mejora en el proceso productivo, aumento de la capacidad de inversión, sinergias con otras empresas, etc.); 0 la provisión de nuevos servicios a la comunidad (ej. disponer de instalaciones dónde llevar residuos metálicos).

En tercer lugar aparecen los impactos sobre la salud y el bienestar psicofísico, con los que se identifica el potencial del proyecto evaluado como generador de enfermedades y perjuicios para la salud física y psíquica de las personas. Se señalan 
así impactos sobre la generación de enfermedades 0 sobre la seguridad de las personas (riesgo de incendio, seguridad vial, etc.). En algunos casos, estos impactos se solapan con otros ya recogidos durante el estudio de los impactos sobre la calidad de vida y el entorno, especialmente aquellos relacionados con contaminación acústica y atmosférica. En este sentido, se han codificado como impactos sobre la salud sólo aquellos fragmentos de texto en los que se hacía referencia explícita a la cuestión sanitaria.

En cuarto lugar se identifican 2 impactos de índole cultural. La escasa aparición de impactos de este tipo no significa que no se hayan valorado cuestiones culturales en el proceso de redacción del EsIA. En la mayoría de documentos se ha dedicado algún espacio a indicar la repercusión del proyecto sobre el patrimonio cultural. Debe señalarse que se ha observado en prácticamente la totalidad de la muestra de EsIA una clara identificación por parte de Ios equipos técnicos de los elementos culturales con los elementos patrimoniales arqueológicos 0 arquitectónicos. En este sentido, no aparecen alusiones a la incidencia del proyecto sobre valores culturales 0 simbólicos inmateriales. En la práctica totalidad de los casos, el estudio de la incidencia cultural del proyecto resulta en la afirmación de que no se han detectado impactos directos sobre el patrimonio histórico 0 arqueológico de la zona. Del mismo modo, se reconoce únicamente como patrimonio cultural aquél que esté inventariado en algún catálogo oficial (Bienes de Interés Cultural, yacimientos arqueológicos registrados, etc.). En relación a los EsIA en los que, efectivamente se detectan impactos culturales por parte de los proyectos evaluados, en un caso se identifica la afectación del proyecto sobre una ruta de uso ciclo-turístico y un área recreativa y, en otro, se aclara que el proyecto se desarrolla en un "Bien Inmueble Etnológico", por lo que se asegurará su conservación y mantenimiento.

No se identifican en ningún caso impactos sobre las estructuras y relaciones sociales de género, familiares y/o comunitarias. Si bien esto podría interpretarse como la ausencia de impactos en este sentido, todo parece indicar que su ausencia refleja más bien la falta de atención por parte de los equipos técnicos a estos elementos de evaluación. Tal y como se ha indicado anteriormente, a pesar de que en la mayoría de los EsIA no se detectan impactos sobre el patrimonio arqueológico, en prácticamente todos los casos se reseña que éstos no existen, manifestándose así que dichos elementos sí han sido considerados durante la fase de evaluación. Lo mismo ocurre con otras áreas de evaluación, como la contaminación acústica 0 atmosférica, para las que en ocasiones se detallan cuales podrían ser sus consecuencias sobre la salud de las personas para, posteriormente, indicar que el proyecto estudiado no tendrá consecuencias en este sentido. Por lo tanto, la falta de mención alguna a la incidencia que sobre las relaciones, dinámicas y estructuras sociales pueden tener los proyectos parece denotar más bien que estos elementos se han ignorado u obviado durante la elaboración del informe.

Tampoco para el caso de los impactos positivos, como ya ocurría en el caso de los procesos de cambio social, se ha identificado en ningún caso que se cumpla lo dispuesto en el Anexo IV de la Ley 21/2013, relativo al acuerdo entre equipo técnico y comunidades afectadas en relación a la valoración de un impacto como positivo.

En relación al aspecto metodológico, en el desarrollo de las labores de identificación de procesos/ impactos sociales observamos un leve incremento en la diversidad de herramientas de recogida de datos, en comparación a lo expuesto para el apartado de inventario socioeconómico. De cualquier modo, se observa una abrumadora presencia de impactos para los cuales no se especifica el procedimiento utilizado para su detección. Esto ocurre en 304 ocasiones a lo largo de los documentos analizados, lo que da cuenta de una evidente ausencia de transparencia y fiabilidad de la información recogida en estos informes. En relación a las herramientas más empleadas, tal y como se muestra en la Tabla 7, la documentación se sitúa en primer lugar, seguida por las mediciones técnicas (mediciones acústicas, de calidad del aire, etc.). El elemento más novedoso se detecta en la alusión a la realización de un grupo de discusión, si bien la información que sobre él se aporta es prácticamente nula, por lo que no es posible valorar su calidad metodológica o de resultados. De cualquier modo, parece intuirse en la referencia a esta técnica clásica de la Sociología que su uso no tenía una finalidad de análisis 
El tratamiento de la dimensión social en los estudios de impacto ambiental: Análisis de contenido aplicado al caso...

de discurso, sino más bien de puesta en común de opiniones de los miembros del equipo de investigación para acordar las puntuaciones otorgadas a cada impacto. En este sentido, y en referencia a la valoración de impactos a través de una matriz de Leopold, se indica:

Dicha valoración, dada la relativa subjetividad del estudio se ha realizado mediante un equipo pluridisciplinar (grupo de discusión) para contrastar y valorar los resultados obtenidos de manera conjunta e individual, cuyo resultado se ha considerado como bases para la realización del mismo.

No queda claro si tal referencia significa que para la realización de la matriz se ha contado con la participación de personas ajenas al equipo de trabajo 0 si, por equipo pluridisciplinar, se refieren al compuesto por el propio equipo redactor (en este caso, formado por dos ingenieros y un biólogo).

Tan solo en 7 ocasiones se hace referencia explícita a que la información que sustenta la identificación o valoración del impacto se ha obtenido a través del contacto directo con la zona de estudio, si bien en ningún caso se refiere a la interacción 0 consulta directa a miembros de la comunidad afectados por el proyecto.

Tabla 7. Herramientas de recogida de información para la identificación de Procesos/Impactos Sociales en el apartado de Identificación y Valoración de Impactos

\begin{tabular}{lc}
\hline & Recuento de citas \\
\hline HERRAM: datos estad. secundarios & 4 \\
\hline HERRAM: documentación & 34 \\
\hline HERRAM: grupo discusión & 1 \\
\hline HERRAM: mediciones téc. & 24 \\
\hline HERRAM: observación directa & 7 \\
\hline HERRAM: Sin especificar & 304 \\
\hline
\end{tabular}

Parte de la explicación a algunas de las cuestiones expuestas en los párrafos anteriores puede encontrarse en la ausencia en los equipos redactores de los EsIA analizados de expertos en el manejo de los conceptos y herramientas propios de las Ciencias Sociales. En algunas ocasiones, el docu- mento de EsIA no especificaba la formación del/los firmante/s. A partir de aquellos documentos en los que sí que se detallaba esta cuestión, se ha podido hacer una relación de las áreas profesionales de los responsables del trabajo evaluado, tal y como se refleja en la Tabla 8 . Se observa una evidente preeminencia de personal especializado en diversas ramas de las Ciencias Naturales, siendo los profesionales de la Ingeniería —especialmente ingeniería industrial—y de la Biología los más frecuentes en estos equipos. Tan solo se han detectado dos EsIA con la presencia de una licenciada en Ciencias Políticas (ambos EsIA, desarrollados por el mismo equipo técnico, por lo que el recuento aparece duplicado en la Tabla 8).

Tabla 8. Área de especialización profesional de los miembros de los equipos de trabajo

\begin{tabular}{lc}
\hline Área de especialización profesional & Recuento \\
\hline Arqueólogo & 1 \\
\hline Arquitecto & 1 \\
\hline Ingeniero/a agroalimentario y medio rural & 1 \\
\hline Ingeniero/a aeronáutico/a & 1 \\
\hline Ingeniero/a agrónomo/a & 16 \\
\hline Ingeniero/a caminos, canales y puertos & 4 \\
\hline Ingeniero/a de minas & 1 \\
\hline Ingeniero/a técnico forestal & 1 \\
\hline Ingeniero/a técnico industrial & 18 \\
\hline Biólogo/a & 18 \\
\hline Ambientólogo/a & 4 \\
\hline Ambientólogo/a e ingeniero/a industrial & 1 \\
\hline Geólogo/a & 2 \\
\hline Politólogo/a & 2 \\
\hline Químico/a & 1 \\
\hline Meteorólogo/a & 1 \\
\hline
\end{tabular}

Con el objetivo de aproximarnos al tipo de trabajo que realizan estos equipos técnicos en términos de diversidad disciplinar, se han contabilizado los documentos de EsIA en función del número de miembros que componen los equipos. Destaca el hecho de que en 38 de los 51 documentos tan solo 
aparece una persona como firmante del texto. Ello explica la ausencia de profundización en el análisis de elementos sociales y las debilidades de los documentos estudiados a la hora de aportar evaluaciones que consideren las relaciones complejas entre entorno físico y social. Únicamente en cuatro ocasiones se han detectado equipos de trabajo que superasen los 2 miembros y tan solo un equipo (encargado de la elaboración de dos EsIA de la muestra analizada) contaba con 7 miembros y un verdadero carácter multidisciplinar.

Tabla 9. Número de miembros de los equipos de trabajo firmantes de los EsIA

\begin{tabular}{lc}
\hline N. $^{\circ}$ de miembros del equipo & $\mathbf{N}^{0}$ de EsIA \\
\hline 1 miembro & 38 \\
\hline 2 miembros & 9 \\
\hline 3 miembros & 1 \\
\hline 7 miembros & 2 \\
\hline No se especifica el equipo & 1 \\
\hline Total EsIA & 51 \\
\hline
\end{tabular}

\section{CONCLUSIONES}

Las conclusiones del análisis realizado no ofrecen una perspectiva optimista en relación a la existencia de avances constatables en la incorporación del análisis de la dimensión social a las Evaluaciones de Impacto Ambiental. Según se desprende de el estudio aquí expuesto, no han surtido efecto significativo las insistentes demandas que por parte de las Ciencias Sociales - y, en concreto, desde los equipos que desde hace años desarrollan herramientas como la Evaluación de Impacto Social—en relación a la necesidad de incorporar a las tareas de evaluación un enfoque que reconozca la compleja interacción entre entorno físico y social. Sirva, por tanto, este trabajo para constatar, con datos actualizados y detallados, la persistencia de todas y cada una de las carencias que ya fueron señaladas por Pardo (1994) hace más de veinte años. Si entonces el diagnóstico realizado por la autora podía achacarse a lo incipiente del procedimiento EIA en nuestro país y a la falta de experiencia y recorrido de los equipos técnicos y de la administración, tales argumentos no resultan válidos en la actualidad y convierten el problema en más grave si cabe.

Los resultados aquí expuestos muestran que, las escasas referencias a elementos sociales se realizan con un elevado nivel de superficialidad y falta de transparencia $y / 0$ calidad metodológica, lo que convierte en irrelevantes los resultados y en poco útil al procedimiento. La ausencia de consultas directas a las comunidades potencialmente afectadas por los proyectos, así como de atención a elementos de índole social que vayan más allá de la mera descripción de las condiciones demográficas, la creación de puestos de trabajo o la generación de impacto acústico - y que en muchas ocasiones tampoco cuentan con un correcto tratamiento-, junto a una inexistente participación ciudadana más allá del proceso de alegaciones, continúan poniendo en cuestión la calidad de las EIA en nuestro país.

Parte del problema podría atribuirse a la existencia de una normativa laxa o ambigua en su exigencia de incorporación de lo social y del empleo de estrategias participativas (a pesar de que la participación se identifique como uno de los principios rectores del procedimiento EIA en la legislación vigente). Sin embargo, tampoco en los pocos casos en los que la norma es más explícita se observa una mejora en la atención a la dimensión social o participativa. Como se ha constatado, en ninguno de los 51 Estudios de Impacto Social analizados, ni en ninguno de los casos en los que en ellos se identifican y valoran los impactos sociales positivos, se cumple el requisito establecido en el Anexo IV de la ley vigente según el cual éstos deberán ser valorados como positivos tanto por la comunidad técnico-científica como por la población en general. Se observa por tanto un claro incumplimiento de la legislación que, al parecer, no obtiene ningún tipo de atención por parte de los organismos a que compete la evaluación de los EsIA. Urge, por tanto, que las administraciones públicas velen por el cumplimiento de las ya escasas exigencias en materia social de las EIA y que actúen como promotoras y garantes de la incorporación de mejoras en este sentido. Sólo a partir de unas directrices más claras por parte de la normativa actual será posible que los equipos técnicos incorporen al proceso aná- 
lisis sociales de mayor calado. A este respecto, cabe señalar también la ausencia de neutralidad técnica que se detecta en numerosas ocasiones en los EsIA. El hecho de que los EsIA los realicen equipos técnicos contratados por el promotor del proyecto impide garantizar la identificación y correcta valoración de impactos que vayan en contra de los intereses privados de las empresas promotoras. La administración pública y, por extensión, la sociedad civil han de asumir su papel de vigilantes de la calidad y utilidad de un procedimiento que, tal y como se desarrolla en la actualidad, no está cumpliendo con el propósito para el que nació. Al menos no, tal y como ha puesto de manifiesto este trabajo, en lo relativo a la identificación y prevención de impactos sobre las poblaciones humanas.

Por último, esperamos que este trabajo pueda servir de elemento de reflexión para los científicos sociales, especialmente aquellos especializados en el área de la Sociología Ambiental, en relación al papel que jugamos a la hora de poner en valor nuestro trabajo y visibilizar la relevancia del mismo para mejorar las tareas aplicadas de gestión y evaluación ambiental. Si algo se desprende con claridad del análisis realizado es la inexistente consideración por parte de promotores, técnicos y Administración respecto del papel y conocimiento teórico y técnico del sociólogo. Parece claro que las múltiples demandas que desde la Sociología Ambiental académica se vienen realizando desde hace décadas están teniendo poco 0 ningún calado en esta práctica profesional. Si bien contamos con múltiples propuestas teóricas en este sentido, como las de la ciencia unificada (Wilson, 1999) o la ciencia trans-disciplinar (Lang et al., 2012), éstas no han conseguido abrirse paso en el ámbito aplicado cotidiano. Urge, por tanto, la búsqueda de vías que permitan superar las fronteras existentes entre la academia y el mundo profesional si deseamos que nuestras contribuciones tengan visos de aplicabilidad.

\section{AGRADECIMIENTOS}

Deseo agradecer la inestimable colaboración de Natividad Albert, así como de Roberto Martínez y Sara Mira en las tareas de codificación y análisis documental que han fundamentado este trabajo. Asimismo, agradezco al Servicio de Evaluación de Impacto Ambiental de la Generalitat Valenciana las facilidades de acceso a la documentación técnica de los expedientes EIA.

Este trabajo es resultado del proyecto "Gestión Social del Medio Ambiente: diagnóstico, oportunidades y resistencias hacia la transdisciplinariedad" (GV/2015/124 - GR-14-14) financiado mediante convocatoria pública por la Generalitat Valenciana y la Universidad de Alicante.

\section{REFERENCIAS BIBLIOGRÁFICAS}

Aledo, A. Domínguez, J. A. (2001). Sociología Ambiental. Granada: Grupo Editorial Universitario.

Alonso, L. E. (1998). La mirada cualitativa en sociología. Madrid: Fundamentos.

Alonso, L. E. (2013). La sociohermenéutica como programa de investigación en sociología. $A R$ BOR, 189 (761).

Barrow, C. J. (2000). Social impact assessment: An introduction. Oxford: Oxford University Press.

Becker, H. A. (2001). Social impact assessment. European Journal of Operational Research, 128 (2), 311-321.

Becker, H. A., Vanclay, F. (2003). The international handbook of social impact assessment: Conceptual and methodological advances. Cheltenham: Edward Elgar Publishing.

Berelson, B. (1952). Content Analysis in Communication Research. Glencoe: Free Press.

Bond, A. J., Morrison-Saunders, A. (2011). Re-evaluating sustainability assessment: Aligning the vision and the practice. Environmental Impact Assessment Review, 31 (1), 1-7.

Bond, R., Curran, J., Kirkpatrick, C., Lee, N., Francis, P. (2001). Integrated impact assessment for sustainable development: A case study approach. World Development, 29 (6), 1011-1024.

Burdge, R. J. (2004). The concepts, process and methods of social impact assessment. Middleton: Social Ecology Press.

Cantó, S., Riera, P. (2003). La vertiente socioeconómica en los estudios de impacto ambiental. Ciudad y Territorio.Estudios Territoriales, 138, 539-550. 
Cashmore, M. (2004). The role of science in environmental impact assessment: Process and procedure versus purpose in the development of theory. Environmental Impact Assessment Review, 24 (4), 403-426.

Clark, B. D. (1994). Improving public participation in environmental impact assessment. Built Environment, 20 (4), 294-308.

Conde, F. (2009). Análisis sociológico del sistema de discursos. Madrid: CIS.

Dendena, B., Corsi, S. (2015). The environmental and social impact assessment: A further step towards an integrated assessment process. Journal of Cleaner Production, 108, 965-977.

Domínguez-Gómez, J. A. (2016). Four conceptual issues to consider in integrating social and environmental factors in risk and impact assessments. Environmental Impact Assessment Review, 56, 113-119.

Echavarren, J. M. (2007). Aspectos socioeconómicos de la evaluación de impacto ambiental. Revista Internacional de Sociología, 65 (47), 99-116.

Esteves, A. M., Franks, D., Vanclay, F. (2012). Social impact assessment: The state of the art. Impact Assessment and Project Appraisal, 30 (1), 34-42.

García, E. (2004). Medio Ambiente y Sociedad: la civilización industrial y los límites del planeta. Madrid: Alianza Editorial.

Glucker, A. N., Driessen, P. P. J., Kolhoff, A., Runhaar, H. A. C. (2013). Public participation in environmental impact assessment: Why, who and how? Environmental Impact Assessment Review, 43, 104-111.

Hartley, N., Wood, C. (2005). Public participation in environmental impact assessment-implementing the aarhus convention. Environmental Impact Assessment Review, 25 (4), 319-340.

Hellström, T., Jacob, M. (1996). Uncertainty and values: The case of environmental impact assessment. Knowledge and Policy, 9 (1), 70-84.

Krippendorf, K. (1980). Content Analysis: An Introduction to Its Methodology. Beverly Hills: SAGE Publications.

Lang, D. J., Wiek, A., Bergmann, M., Stauffacher, M., Martens, P., Moll, P. (2012). Transdisciplinary research in sustainability science: Practice, principles, and challenges. Sustainability Science, 7 (1), 25-43.

Lee, B., Haworth, L., Brunk, C. G. (1995). Values and science in impact assessment. Environments, 23 (1), 93-100.

Lee, N. (2006). Bridging the gap between theory and practice in integrated assessment. Environmental Impact Assessment Review, 26 (1), 57-78.

López, I., Arriaga, A., Pardo, M. (2018). La dimensión social del concepto de desarrollo sostenible: ¿La eterna olvidada? Revista Española de Sociología, 27 (1), 25-41.

Momtaz, S. (2006). Public participation and community involvement in environmental and social impact assessment in developing countries. The International Journal of Environmental, Cultural, Economic \& Social Sustainability, 2, 89-97.

Momtaz, S., Kabir, S. Z. (2013). Evaluating environmental and social impact assessment in developing countries. Waltham: Elsevier.

Morrison-Saunders, A., Arts, J. (2005). Learning from experience: Emerging trends in environmental impact assessment follow-up. Impact Assessment and Project Appraisal, 23 (3), 170-174.

O'Faircheallaigh, C. (2010). Public participation and environmental impact assessment: Purposes, implications, and lessons for public policy making. Environmental Impact Assessment Review, 30 (1), 19-27.

Palerm, J. R. (2000). An empirical-theoretical analysis framework for public participation in environmental impact assessment. Journal of Environmental Planning and Management, 43 (5), 581-600.

Pardo, M. (1994). El impacto social en las evaluaciones de impacto ambiental: Su conceptualización y práctica. Revista Española de Investigaciones Sociológicas, 66, 141-167.

Pardo, M. (1997). Environmental impact assessment: Myth or reality? lessons from spain. Environmental Impact Assessment Review, 17 (2), 123-142. Pope, J., Annandale, D., Morrison-Saunders, A. (2004). Conceptualising sustainability assessment. Environmental Impact Assessment Review, 24 (6), 595-616. 
Rossini, F. A., Porter, A. L. (1983). Integrated impact assessment. Boulder: Westview Press, Inc.

Salomons, G. H., Hoberg, G. (2014). Setting boundaries of participation in environmental impact assessment. Environmental Impact Assessment Review, 45, 69-75.

Tilleman, W. A. (1995). Public participation in the environmental impact assessment process: A comparative study of impact assessment in canada, the united states and the european community. Columbia Journal of Transnational Law, 33 (2), 337-340.

Torriti, J. (2011). The unsustainable rationality of impact assessment. European Journal of Law and Economics, 31 (3), 307-320.

Vanclay, F. (2002). Conceptualising social impacts. Environmental Impact Assessment Review, 22 (3), 183-211.
Vanclay, F. (2003). International principles for social impact assessment. Impact Assessment and Project Appraisal, 21 (1), 5-12.

Vanclay, F., Bronstein, D. A. (1995). Environmental and social impact assessment. Chichester: John Wiley \& Sons.

Vanclay, F., Esteves, A. M. (2011). New directions in social impact assessment: Conceptual and methodological advances. Cheltenham: Edward Elgar Publishing.

Wilkins, H. (2003). The need for subjectivity in ElA: Discourse as a tool for sustainable development. Environmental Impact Assessment Review, 23 (4), 401-414.

Wilson, E. 0. (1999). Consilience: la unidad del conocimiento. Barcelona: Galaxia GutenbergCírculo de Lectores. 
